\title{
Lumbar decompression in the elderly: increased age as a risk factor for complications and nonhome discharge
}

\author{
Meghan E. Murphy, MD,1,2 Hannah Gilder, BS, 1,2 Patrick R. Maloney, MD,,2 \\ Brandon A. McCutcheon, MD, MPP, 1,2 Lorenzo Rinaldo, MD, PhD, ${ }^{1,2}$ Daniel Shepherd, MD, ${ }^{1,2}$ \\ Panagiotis Kerezoudis, MD,, Daniel S. Ubl, MPH, ${ }^{3}$ Cynthia S. Crowson, MS, ${ }^{3}$ \\ William E. Krauss, MD, ${ }^{1}$ Elizabeth B. Habermann, PhD, MPH, ${ }^{3}$ and Mohamad Bydon, MD $^{1,2}$

\begin{abstract}
${ }^{1}$ Department of Neurologic Surgery, Mayo Clinic; ${ }^{2}$ Mayo Clinic Neuro-Informatics Laboratory; and ${ }^{3}$ Department of Health Sciences Research, Mayo Clinic, Rochester, Minnesota
\end{abstract}

\begin{abstract}
OBJECTIVE With improving medical therapies for chronic conditions, elderly patients increasingly present as candidates for operative intervention for degenerative diseases of the spine. To date, there is a paucity of studies examining complications in lumbar decompression, without fusion, that include patients older than 80 years. Using a multicenter national database, the authors of this study evaluated lumbar decompression in the elderly, including octogenarians, to evaluate for associations between age and patient outcomes.
\end{abstract}

METHODS The 2011-2013 American College of Surgeons' National Surgical Quality Improvement Program data set was queried for patients 65 years and older with diagnosis and procedure codes inclusive of degenerative spine disease and lumbar decompression without fusion. Morbidity and mortality within the 30-day postoperative period were the primary outcomes. Secondary outcomes of interest included unplanned readmission within 30 days or discharge to a nonhome facility. Outcomes and operative characteristics were compared using chi-square tests, Kruskal-Wallis tests, and multivariable logistic regression models.

RESULTS A total of 8744 patients were identified; of these patients $4573(52.30 \%)$ were 65 years and older. Elderly patients were stratified into 3 age categories: 85 years or older $(n=314), 75-84$ years $(n=1663)$, and $65-74$ years $(n=$ 2596). Univariate analysis showed that, compared with age younger than 65 years, increased age was associated with the number of levels $(\geq 3)$, readmissions within 30 days, nonhome discharge, any complication, length of stay, and blood transfusion (all $p<0.001)$. On multivariable analysis and with younger than 65 years as the reference, increased age was associated with any minor complication ( $p<0.001$; $\geq 85$ years: OR 3.47, 95\% Cl 1.69-7.13; 75-84 years: OR 2.34, 95\% $\mathrm{Cl} 1.45-3.78$; and 65-74 years: OR 1.44, 95\% Cl 0.94-2.20), as well as discharge location other than home $(p<0.001$; $\geq 85$ years: OR $13.59,95 \%$ Cl 9.47-19.49; 75-84 years: OR 5.64, 95\% Cl 4.33-7.34; and 65-74 years: OR 2.61, 95\% Cl 2.05-3.32).

CONCLUSIONS The authors' high-powered, multicenter analysis of lumbar decompression without fusion in the elderly, specifically including patients older than 80 years, demonstrates that increased age is associated with more extensive operations, resulting in longer hospital stays, increased rates of nonhome discharge, and minor complications.

https://thejns.org/doi/abs/10.3171/2016.8.SPINE16616

KEY WORDS lumbar decompression; elderly; degenerative spine disease; postoperative complications; NSQIP

ABBREVIATIONS ACS-NSQIP = American College of Surgeons' National Surgical Quality Improvement Program; ASA = American Society of Anesthesiologists; $B$ MI = body mass index; $\mathrm{BUN}=$ blood urea nitrogen; $\mathrm{CHF}=$ congestive heart failure; $\mathrm{CKD}=$ chronic kidney disease; $\mathrm{COPD}=$ chronic obstructive pulmonary disease; $\mathrm{CPT}=\mathrm{Cur}-$ rent Procedural Terminology; DVT = deep vein thrombosis; eGFR = estimated GFR; GFR = glomular filtration rate; INR = international normalized ratio; IQR = interquartile range; $\mathrm{SSI}=$ surgical site infection; UTI = urinary tract infection.

SUBMITTED May 26, 2016. ACCEPTED August 3, 2016.

INCLUDE WHEN CITING Published online November 18, 2016; DOI: 10.3171/2016.8.SPINE16616. 
A S average life expectancy increases worldwide, larger populations of elderly patients are drastically increasing their health care utilization, thus challenging health care systems to provide diagnostic and management options that allow for maintenance of functional status and quality of life. In the United States, overall life expectancy has increased from 73.66 to 78.74 years over the last 2 decades, with current averages of 76 years for men and 81 years for women (http://www.who. int/countries/usa/en/). Approximately $75 \%$ of the global population born between the years of 2000 and 2005 is expected to reach the age of 60 years, while $33 \%$ is expected to reach the age of 80 years, with expectations for these proportions to increase in the coming decades. ${ }^{11,29}$ Older patients are more likely to have a higher comorbidity burden, often with multiple systems involved. ${ }^{26}$ The complexity in health status poses unique challenges in achieving balance with therapies and interventions that allow for realization of goals of care, specifically, maintaining functional independence. Conservative therapy fails for some diseases and surgery is considered; degenerative disease of the spine is one of these pathologies.

Degenerative disease of the spine is a widely prevalent and debilitating disease in the elderly. All structures of the spinal column can be affected, including vertebral bodies and posterior elements, intervertebral discs, facet joints, ligamentous structures, and surrounding musculature. ${ }^{11}$ Degenerative pathology is attributed to osteoarthritic changes, spondylolisthesis, degenerative disc disease, facet joint hypertrophy, and ligamentum flavum hypertrophy. Degenerative changes in the lumbar spine can cause chronic low-back pain, sensory disturbance, and motor decline, often leading to recurrent falls and limitations in activities of daily living. ${ }^{27}$ While initial management of degenerative lumbar spine disease includes physical therapy, pharmacotherapy, and epidural steroid injections, symptoms can be refractory to these conservative measures. The natural history of the disease involves progression and worsening symptoms. In up to $60 \%$ of patients, conservative treatment will fail to ameliorate the patient's symptoms, thus necessitating the need to explore surgical options. ${ }^{3}$ Determining surgical candidacy requires careful balancing of the risks with the benefits of functional improvement and quality of life. Long-standing coexisting comorbidities, such as diabetes mellitus, congestive heart failure (CHF), and history of myocardial infarction, can preclude surgical intervention in certain patients, carrying increased risk of complications and poor outcomes. ${ }^{1,6}$ Nevertheless, some literature has shown that lumbar spine surgery in elderly patients leads to good functional results with acceptable risks by decreasing pain and disability, improving quality of life, and minimizing the need for chronic pain medications. . $^{7,8,12,14,23,24}$ However, many of the studies are from single institutions, include small cohorts, or fail to include patients older

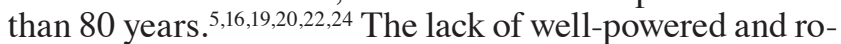
bust outcomes studies on lumbar decompression without fusion in the elderly provides a gap in literature that this study attempts to fill. Using a large national multicenter database, we evaluated morbidity and mortality in elderly patients undergoing lumbar decompression without fusion. Improved characterization of predictive risk factors for this patient population will better inform both patients and surgeons in their preoperative discussions regarding surgical candidacy.

\section{Methods \\ Inclusion and Exclusion Criteria}

This retrospective analysis used the American College of Surgeons' National Surgical Quality Improvement Program (ACS-NSQIP) Participant Use Data File to define and abstract data for our cohort. More than 250 variables, including preoperative health status markers, intraoperative data, and 30-day postoperative morbidity and mortality outcomes, are recorded for each surgical patient record submitted from participating sites. Specialized training occurs for employees who abstract data at each institution. The quality control processes are highly regulated with interrater reliability audits, ensuring maintenance of a robust data registry with high fidelity. ${ }^{25}$ The ACS-NSQIP website can be accessed for more information regarding details of the registry, recorded data, and participating institutions (https://www.facs.org/quality-programs/acs-nsqip).

Patients were included for analysis if they were enrolled in the NSQIP data registry between 2011 and 2013 and underwent lumbar decompression surgery, as determined by identification of Current Procedural Terminology (CPT) codes $(n=13,915)$. The specified codes included lumbar region laminectomies, foraminotomies, and/or facetectomies at one or more levels for the purpose of decompression of the spinal cord, cauda equina, and/ or nerve roots (CPT codes, 63005, 63012, 63017, 63047 from the American Medical Association Current Procedural Terminology, Professional Edition, 2012). Exclusion criteria and filtering processes for refining our cohort are outlined in Fig. 1. Emergent cases and cases involving infection and neoplastic diagnoses were excluded. Cases with prior operation or chemotherapy within 30 days prior to the procedure were also excluded. Cases with incorrect coding (e.g., CPT 63048 [additional vertebral segment, lumbar] without initial procedure CPT 63047 [single vertebral segment, lumbar]) as well as cases with concurrent CPT codes indicating fusion were excluded. This study was exempt from review by our institutional review board because of the de-identified NSQIP data.

\section{Outcomes}

Morbidity and mortality within the 30-day postoperative period were the primary outcomes. Morbidity included the following perioperative complications: requirement for blood transfusion, venous thromboembolism (pulmonary embolism or deep vein thrombosis [DVT]), surgical site infection (SSI), wound dehiscence, septic shock, stroke, nerve injury, cardiac arrest, coma, urinary tract infection (UTI), renal failure, renal insufficiency, failure to wean ventilatory support, reintubation within 48 hours of the operation, and pneumonia. These complications were examined both separately and as an aggregate to capture total complication rates. Minor complications included UTI or superficial SSI, while major complications included cardiac arrest, myocardial infarction, cerebrovascular accidents, deep SSI, wound disruption, sepsis, sep- 


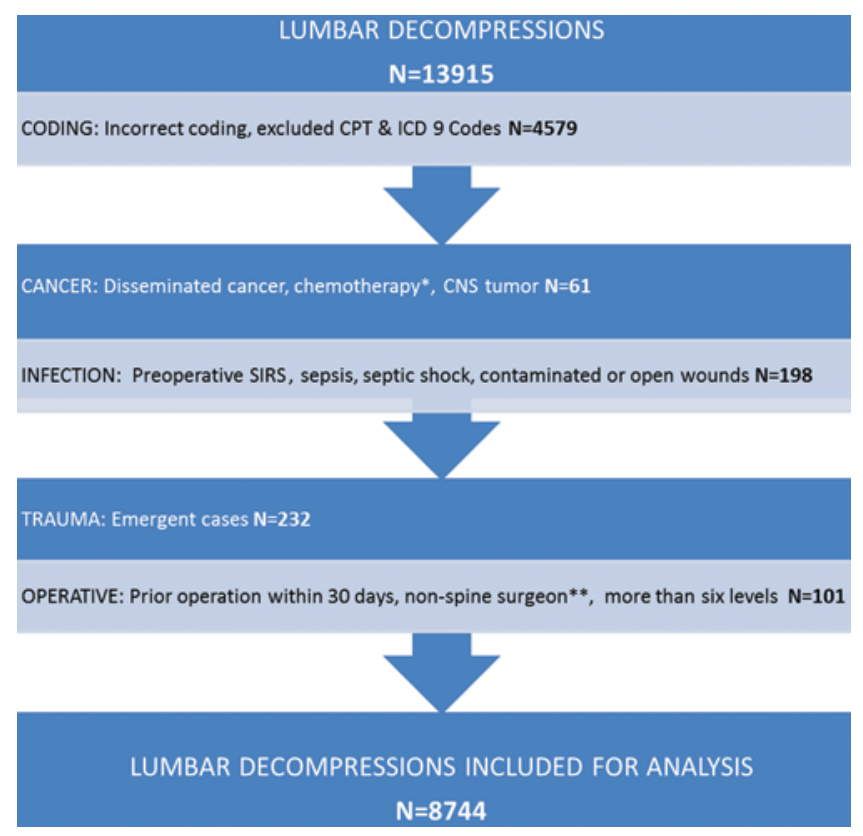

FIG. 1. Methods exclusion flowchart. *Chemotherapy within 30 days of index procedure. ${ }^{* *}$ Non-spine surgeon $=$ specialty other than orthopedics or neurosurgery. SIRS = systemic inflammatory response syndrome. Figure is available in color online only.

tic shock, unplanned reintubation, ventilator dependence exceeding 48 hours after surgery, pneumonia, acute renal failure, venous thromboembolism, and reoperation.

Secondary outcomes of interest included unplanned readmission within 30 days or discharge to a nonhome facility. Further exclusions for the secondary outcomes were required. In regard to readmission, cases without readmission data were excluded $(\mathrm{n}=191)$. For discharge disposition, the following additional exclusions were applied: patients without discharge information $(n=22)$, those admitted from a facility $(n=68)$, those discharged back to their home facility $(n=22)$, and in-hospital death $(n=5)$.

\section{Covariates}

Patient characteristics available for analysis included age, sex, body mass index (BMI), and race/ethnicity. Comorbidities available within the database included hypertension requiring medication, diabetes mellitus, $\mathrm{CHF}$, other cardiac history, chronic obstructive pulmonary disease (COPD), peripheral vascular disease, prior stroke or transient ischemic attack (TIA), and chronic steroid use within 30 days preceding surgery. Lifestyle factors were also available for analysis, including status as a current smoker (defined as current use within the previous year). American Society of Anesthesiologists (ASA) physical status classification was also collected. Preoperative laboratory values of coagulation parameters (prothrombin time, partial thromboplastin time, and international normalized ratio (INR), blood counts (hematocrit, platelet count, and leukocyte count), renal function measurements (glomerular filtration rate [GFR] as calculated by the chronic kidney disease [CKD]-epidemiology formula, blood urea nitrogen [BUN], and serum creatinine), and

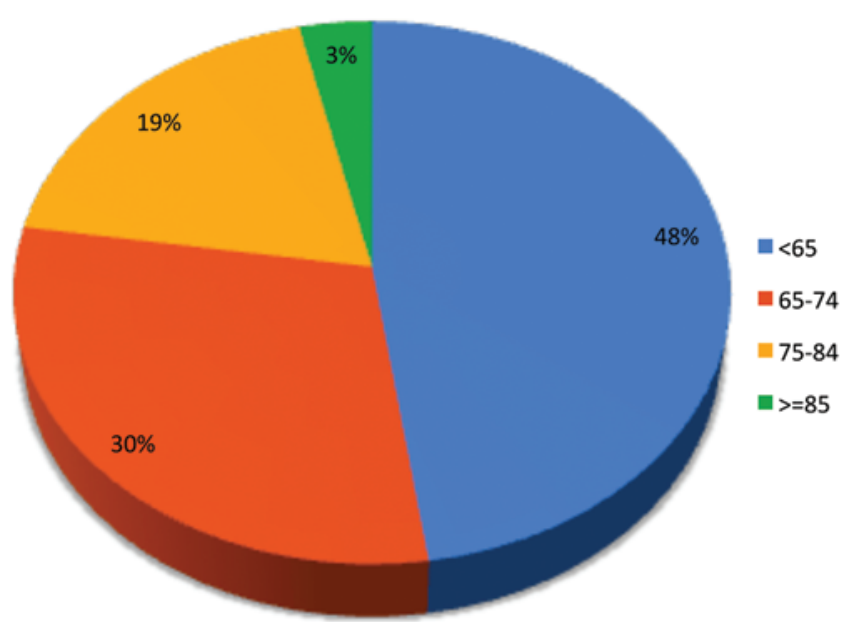

FIG. 2. Distribution of patients across age groups (in years). Figure is available in color online only.

liver function (albumin and alkaline phosphatase) were recorded. Abnormal values were defined as follows: anemia (hematocrit $<35 \%$ ), leukocytosis (white blood cell count $\geq 10,000 / \mu \mathrm{l}$ ), thrombocytopenia (platelet count $<150,000 /$ $\mu \mathrm{l}$ ), liver function decline or malnutrition (albumin $<3.5$ $\mathrm{g} / \mathrm{dl}$ and/or INR > 1.3), and renal function decline (BUN $>33 \mathrm{mg} / \mathrm{dl}$, creatinine $>1 \mathrm{mg} / \mathrm{dl}$, and/or estimated GFR [eGFR] $<90 \mathrm{ml} / \mathrm{min} / 1.73 \mathrm{~m}^{2}$ ). Operative variables including procedure time, number of levels decompressed, and requirement for blood transfusions were also available for study. ${ }^{17}$

\section{Statistical Analysis}

Patient characteristics, demographics, and covariates of interest were studied for overall distribution. Subsequent unadjusted analyses using chi-square, Fisher's exact, and Kruskal-Wallis tests were used to evaluate individual associations with morbidity and mortality. Adjusting for potential confounders, with the threshold for statistical significance at $p=0.05,30$-day outcomes of any minor complication, any major complication, readmission, and discharge to a nonhome facility were assessed using multivariable logistic regression models (SAS version 9.3, SAS Institute Inc.).

\section{Results}

Following data set query and application of inclusion and initial exclusion criteria, 8744 patients were identified for analysis. The median age (interquartile range [IQR]) was $65(56-74)$, and $52.3 \%(n=4573)$ of patients were categorized as 65 years of age or older. Within this cohort, $29.7 \%(\mathrm{n}=2596)$ ranged in age from 65 to 74 years, $19.0 \%$ $(n=1663)$ were 75 to 84 years, and $3.6 \%(n=314)$ were 85 years of age or older (Fig. 2). A vast majority (79.9\%) of patients were Caucasian, and $60.1 \%$ were male. The median BMI (IQR) was 30 (26-34) for all patients. Nearly one-fifth $(17.6 \%)$ of patients were current smokers within 1 year of surgery. The majority of patients were in ASA Class III or better and were functionally independent at the time of surgery ( $97.7 \%$ and $97.5 \%$, respectively). Hy- 
pertension requiring medication was the most common comorbidity present among patients, occurring in $60.4 \%$ of patients. Approximately $6 \%$ of patients with available data were found to be either preoperatively anemic with a hematocrit less than $35 \%$ or thrombocytopenic with a platelet count less than $150,000 / \mu 1$. Approximately onehalf of patients $(50.4 \%)$ were classified as having Stage 2 CKD. Most patients underwent a 1-level (45.8\%) or 2-level $(41.1 \%)$ decompression, and $13.1 \%$ underwent a procedure involving 3 or more levels. The median (IQR) operative time was 98 minutes (70-139 minutes).

Univariate comparisons of patient demographic and preoperative characteristics across age groups are presented in Table 1. A greater percentage of patients 85 and older had lower BMI $\left(\mathrm{kg} / \mathrm{m}^{2}\right)$ in the range of 18.5-24.9 (31.0\%) or 25.0-29.9 (45.2\%) compared with those younger than 65 years, with $15.9 \%$ and $31.3 \%$ of these patients falling into the same respective BMI ranges $(\mathrm{p}<0.001)$. Conversely, a greater proportion of patients younger than 65 were noted to have a BMI denoting Class II or III obesity, with $15.4 \%$ falling within a range of 35-39.9 and $10.2 \%$ having a BMI of 40 or greater. Only $3.2 \%$ and $1.3 \%$ of patients 85 years and older fell into the same respective BMI ranges $(\mathrm{p}<$ 0.001 ). A greater proportion of younger patients were active smokers within 1 year of surgery. In patients younger than 65 years, $27.4 \%$ were smokers, compared with $11.6 \%$ in the 65- to 74-year age group and $3.2 \%$ in the 85 years and older age group $(\mathrm{p}<0.001)$. A greater proportion of older patients were partially or totally functionally dependent, with $7.7 \%$ of those 85 years and older falling into this category, compared with $3.6 \%$ of those $75-84$ years and approximately $2 \%$ for both the 65 - to 74 -year and the younger than 65 -year age groups $(\mathrm{p}<0.001)$.

In regard to comorbidities, older patients more often had histories of pulmonary, cardiac, and vascular disease. Patients younger than 65 years were significantly less likely to have COPD, diabetes mellitus, CHF, hypertension requiring medication, and chronic corticosteroid treatment compared with those 65 years and older (all $p<0.05$ ). Of those patients 85 years and older, $6.1 \%$ were classified as ASA Class IV, compared with $4.6 \%, 2.4 \%$, and $1.0 \%$ for the 75- to 84-year, 65- to 74-year, and younger than 65year groups (all $\mathrm{p}<0.001$ ).

Several preoperative laboratory values indicative of end-organ dysfunction were significantly associated with advanced patient age. Anemia, leukocytosis, thrombocytopenia, liver function decline or malnutrition, and renal function decline occurred in a significantly smaller percentage of patients younger than 65 years compared with those 85 years and older $(\mathrm{p}<0.001)$. There was a significant association between the number of levels of decompression and age. Of patients undergoing singlelevel decompressions, the majority were younger than 65 years (52.0\%), 2-level decompressions were most common among the 75 - to 84 -year age group (44.9\%), and 3 or more levels were most often performed in patients 85 years and older $(21.3 \%)$ ( $\mathrm{p}<0.001)$. While the difference in median operative time was statistically significant across age groups, the maximum difference of only 8.5 minutes was not clinically significant.

Univariate analyses in regard to outcomes of interest are detailed in Table 2 . The overall mortality rate within 30 days was $0.2 \%$, with $35.3 \%$ of these deaths occurring in the hospital. The overall rate of postoperative complications was $5.3 \%$. Patients in older age groups were sequentially more likely to develop complications, with significantly higher rates in patients 85 years and older compared with those younger than 65 years $(7.3 \%$ vs $4.1 \%, \mathrm{p}<0.001)$. The highest rates of both minor and major complications occurred in patients 75 years and older. Of the patients 85 years and older, $4.1 \%$ of them developed a minor complication while $4.5 \%$ developed a major complication compared with $1.4 \%$ and $3.0 \%$, respectively, for those patients younger than 65 years old (all p $<0.01$ ). Patients aged 65-74 years, 75-84 years, and 85 years and older had higher percentages of DVT at $0.7 \%, 0.7 \%$, and $0.6 \%$, respectively, compared with the $0.2 \%$ of those younger than 65 years $(\mathrm{p}=0.015)$. Patients 85 years and older received more blood transfusions than those younger than 65 years $(3.8 \%$ vs $1.2 \%$, p < 0.001$)$, with transfusion rates increasing sequentially with advancing age. $\mathrm{Pa}$ tients 85 years and older experienced significantly longer total hospital stays than those younger than 65 years with a median (IQR) of 2 days (1-4 days) compared with 1 day (1-2 days) $(\mathrm{p}<0.001)$. Older age was not associated with increased rates of reoperation. Discharge to a facility other than home increased sequentially with advancing age, with a significantly greater proportion $(34.2 \%)$ of patients 85 years and older being discharged to a nonhome facility, compared with $3.7 \%$ of patients younger than 65 years $(\mathrm{p}<0.001)$.

The association between age and postoperative outcomes was investigated in a risk-adjusted manner using multivariable logistic regression analysis controlling for the following patient characteristics, comorbidities, laboratory values, and operative variables: race, sex, BMI, hypertension, diabetes, COPD, functional status, smoking status, GFR, hematocrit, platelet count, INR, ASA class, number of levels, and operative time (Table 3). Patients 75-84 years (OR 2.34, 95\% CI 1.45-3.78) and those 85 years and older (OR 3.47, 95\% CI 1.69-7.13) were significantly more likely than patients younger than 65 years to develop a minor complication following surgery. Older age was significantly associated with an increased likelihood of discharge to a facility other than home when compared with patients younger than 65 years: 65 to 74 years, OR 2.61 (95\% CI 2.05-3.12); 75-84 years, OR 5.64 (95\% CI 4.33-7.34); and 85 and older, OR 13.59 (95\% CI 9.47-19.49). Readmission and major complications were not found to be associated with age.

\section{Discussion}

The increasing population of the elderly influences patient demographics, pathological variation, and clinical presentations evaluated by spine surgeons. ${ }^{11}$ Published literature has posed conflicting evidence on the risks and benefits of lumbar decompression surgery in elderly patients. Increased patient age has previously correlated with poor postoperative outcomes, including prolonged length of stay in the hospital, increased postsurgical complications, adverse discharge, and limited improvement in pre- 
TABLE 1. Patient characteristics, comorbidities, laboratory values, and operative data across age groups

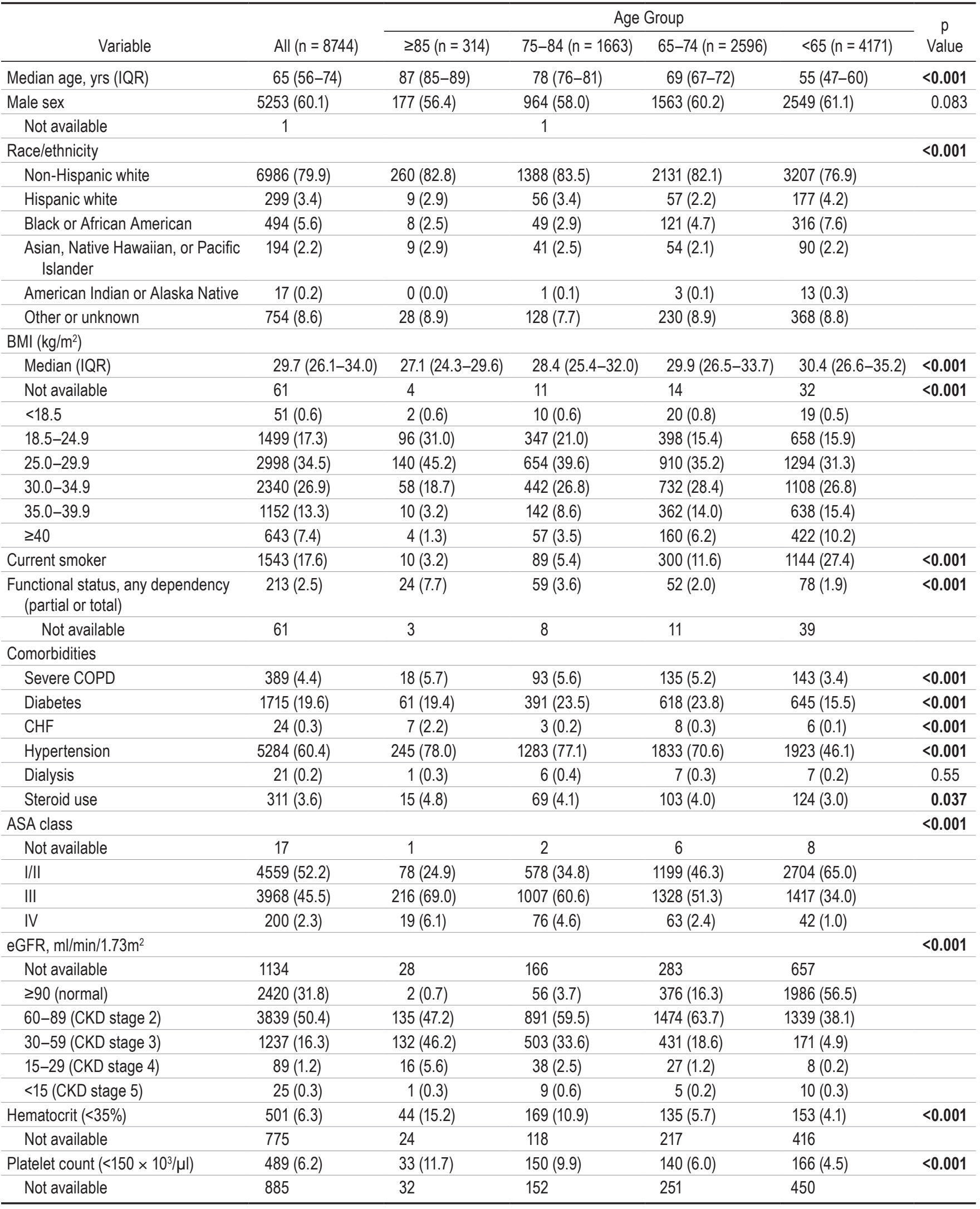


» CONTINUED FROM PAGE 357

TABLE 1. Patient characteristics, comorbidities, laboratory values, and operative data across age groups

\begin{tabular}{|c|c|c|c|c|c|c|}
\hline \multirow[b]{2}{*}{ Variable } & \multirow[b]{2}{*}{ All $(n=8744)$} & \multicolumn{4}{|c|}{ Age Group } & \multirow{2}{*}{$\begin{array}{c}p \\
\text { Value }\end{array}$} \\
\hline & & $\geq 85(n=314)$ & $75-84(n=1663)$ & $65-74(n=2596)$ & $<65(n=4171)$ & \\
\hline INR ( $\geq 1.3)$ & $100(1.9)$ & $5(2.6)$ & $29(2.9)$ & $39(2.6)$ & $27(1.1)$ & $<0.001$ \\
\hline Not available & 3548 & 125 & 661 & 1097 & 1665 & \\
\hline Albumin $(<3.5 \mathrm{~g} / \mathrm{dl})$ & $176(5.4)$ & 17 (12.8) & $54(8.1)$ & $52(5.5)$ & $53(3.6)$ & $<0.001$ \\
\hline Not available & 5510 & 181 & 994 & 1642 & 2693 & \\
\hline WBC $\left(\geq 10 \times 10^{3} / \mu \mathrm{l}\right)$ & 894 (11.4) & $27(9.6)$ & $130(8.6)$ & $233(9.9)$ & $504(13.6)$ & $<0.001$ \\
\hline Not available & 894 & 33 & 152 & 254 & 455 & \\
\hline BUN ( $\geq 33 \mathrm{mg} / \mathrm{dl})$ & $249(3.4)$ & $22(8.0)$ & $105(7.3)$ & $84(3.8)$ & 38 (1.1) & $<0.001$ \\
\hline Not available & 1397 & 39 & 228 & 388 & 742 & \\
\hline Creatinine (>1.0 mg/dl) & $2339(30.7)$ & $129(45.1)$ & $653(43.6)$ & $788(34.1)$ & 769 (21.9) & $<0.001$ \\
\hline Not available & 1133 & 28 & 165 & 283 & 657 & \\
\hline No. of levels & & & & & & $<0.001$ \\
\hline 1 & $4001(45.8)$ & 117 (37.3) & $618(37.2)$ & $1096(42.2)$ & $2170(52.0)$ & \\
\hline 2 & 3595 (41.1) & $130(41.4)$ & $747(44.9)$ & $1129(43.5)$ & 1589 (38.1) & \\
\hline$\geq 3$ & 1148 (13.1) & $67(21.3)$ & $298(17.9)$ & $371(14.3)$ & $412(9.9)$ & \\
\hline Median total op time, in mins (IQR) & $98(70-139)$ & $97(74-135)$ & $103(74-143)$ & $100(71-143)$ & $94.5(68-134)$ & $<0.001$ \\
\hline
\end{tabular}

ASA class $=$ ASA Physical Status Classification; $\mathrm{WBC}=$ white blood cell.

Boldface type indicates statistical significance.

Lumbar decompression: cases with primary CPT for laminectomy (Codes 63005, 63012, 63017, 63047).

operative functional status., ${ }^{1,4,6,9,13}$ Age may serve as a surrogate for progressive systemic pathology and dysfunction, and increased age may lead to physician bias in pursuing conservative treatment. ${ }^{26,27}$ Recent studies have reaffirmed the findings of increased length of stay, increased risk of major postoperative medical complications, and a greater likelihood of nonroutine discharge in elderly patients undergoing lumbar spine surgery., ${ }^{9,13}$ However, evidence also exists that lumbar decompression surgery may be performed safely and efficiently without undue risk to the elderly patient, and may in fact result in superior patient outcomes when compared with conservative treatment. . $^{2,5,7,21,22}$

The majority of patients in our cohort were classified as elderly, based on an age cutoff of 65 years. Although 65 years is a commonly set threshold, given eligibility of Medicare benefits, we found it useful for the purposes of our study to further categorize patients into groups (65-74 years, $75-84$ years, and 85 years and older). This allowed for the observation of data trends across groups of increasing age, as well as direct comparison between any given elderly age group and patients younger than 65 years. The limitations of prior studies include the low numbers or complete omission of patients older than 80 years in the analysis. ${ }^{24}$ In studies including patients older than 80 years, the cohorts were often small, representing surgical cases from a single institution, or included fusions in the operative cohort..$^{416}$ Of 2 multicenter studies, one included 46 patients 80 years and older while the other included 105 patients 80 years and older; this is in comparison with our cohort of 314 patients 85 years and older as well as 1663 patients $75-84$ years. ${ }^{19,22}$ The 46 patients 80 years and older were a part of a total cohort of 241 patients undergoing decompressions only, the remaining 195 patients were younger than 80 years old; no significant difference was found between age groups with respect to postoperative complications. ${ }^{22}$ The SPORT (Spine Patient Outcomes Research Trial) included both decompression and fusion in the surgical cohort and compared outcomes with conservative management. In the SPORT, 58 of the 105 patients in the group of 80 years and older and 749 of the 1130 patients younger than 80 years underwent lumbar spine surgery, with operative management including arthrodesis as an adjunct to decompression in cases with spondylolisthesis; no significant difference in complications was observed between age groups..$^{22}$ The use of the ACS-NSQIP database addresses these limitations by providing patient information for a large cohort of individuals across a wide spectrum of ages, including very elderly patients 85 years and older. As part of a multicenter database, patients in this analysis also represent a variety of operative and postoperative protocols that may differ across hospitals and therefore influence outcomes reported from a single center. Using selective codes for cohort refinement, our analysis also excludes patients undergoing fusions, isolating the lumbar decompression population. Overall, this analysis represents a high-powered study of elderly patients, including octogenarians, undergoing lumbar decompression surgery without fusion in multiple surgical centers within the United States.

Our data reveal that a significantly higher percentages of elderly patient, compared with the cohort younger than 65 years, experienced postoperative complications, required readmission within 30 days of surgery, had prolonged admissions, and were discharged to a nonhome facility. Moreover, the proportion of patients within each age group experiencing certain adverse outcomes increased with increasing age. These adverse outcomes included any complication, adverse discharge, readmission, and 
TABLE 2. Outcomes across age groups: univariate analysis

\begin{tabular}{|c|c|c|c|c|c|c|}
\hline \multirow[b]{2}{*}{ Variable } & \multirow{2}{*}{$\begin{array}{c}\text { All } \\
(n=8744)\end{array}$} & \multicolumn{4}{|c|}{ Age Group (yrs) } & \multirow{2}{*}{$\begin{array}{c}\mathrm{p} \\
\text { Value }\end{array}$} \\
\hline & & $\geq 85(n=314)$ & $75-84(n=1663)$ & $65-74(n=2596)$ & $<65(n=4171)$ & \\
\hline Death w/in 30 days & $17(0.2)$ & $2(0.6)$ & $5(0.3)$ & $3(0.1)$ & $7(0.2)$ & 0.14 \\
\hline In-hospital death & $6(35.3)$ & $0(0.0)$ & $3(60.0)$ & $0(0.0)$ & $3(42.9)$ & 0.24 \\
\hline Not available & 8727 & 312 & 1658 & 2593 & 4164 & \\
\hline Any complication & $460(5.3)$ & $23(7.3)$ & $114(6.9)$ & $150(5.8)$ & $173(4.1)$ & $<0.001$ \\
\hline Any minor complication* & $175(2.0)$ & $13(4.1)$ & $51(3.1)$ & $51(2.0)$ & $60(1.4)$ & $<0.001$ \\
\hline Any major complication† & $330(3.8)$ & $14(4.5)$ & $76(4.6)$ & $113(4.4)$ & $127(3.0)$ & 0.008 \\
\hline Any SSI & $140(1.6)$ & $7(2.2)$ & $28(1.7)$ & $36(1.4)$ & $69(1.7)$ & 0.64 \\
\hline Pneumonia & $27(0.3)$ & $0(0.0)$ & $9(0.5)$ & $14(0.5)$ & $4(0.1)$ & 0.001 \\
\hline DVT/thrombophlebitis & $43(0.5)$ & $2(0.6)$ & $12(0.7)$ & $19(0.7)$ & $10(0.2)$ & 0.015 \\
\hline Pulmonary embolism & $27(0.3)$ & $0(0.0)$ & $7(0.4)$ & $10(0.4)$ & $10(0.2)$ & 0.43 \\
\hline Myocardial infarction & $18(0.2)$ & $2(0.6)$ & $7(0.4)$ & $5(0.2)$ & $4(0.1)$ & 0.021 \\
\hline Cardiac arrest & $11(0.1)$ & $0(0.0)$ & $5(0.3)$ & $1(0.0)$ & $5(0.1)$ & 0.15 \\
\hline Transfusions & $171(2.0)$ & $12(3.8)$ & $45(2.7)$ & $63(2.4)$ & $51(1.2)$ & $<0.001$ \\
\hline UTI & $99(1.1)$ & $10(3.2)$ & $34(2.0)$ & $32(1.2)$ & $23(0.6)$ & $<0.001$ \\
\hline Stroke & $10(0.1)$ & $0(0.0)$ & $3(0.2)$ & $5(0.2)$ & $2(0.0)$ & 0.22 \\
\hline Sepsis or septic shock & $44(0.5)$ & $2(0.6)$ & $13(0.8)$ & $12(0.5)$ & $17(0.4)$ & 0.32 \\
\hline Median hospital LOS in days (IQR) & $1(1-3)$ & $2(1-4)$ & $2(1-3)$ & $2(1-3)$ & $1(1-2)$ & $<0.001$ \\
\hline Return to operating room & $196(2.2)$ & $8(2.5)$ & $37(2.2)$ & $62(2.4)$ & $89(2.1)$ & 0.89 \\
\hline Discharge destination & & & & & & $<0.001$ \\
\hline Not available & 117 & 7 & 29 & 25 & 56 & \\
\hline Home or home facility & $7793(90.3)$ & $202(65.8)$ & $1313(80.4)$ & $2315(90.0)$ & $3963(96.3)$ & \\
\hline $\begin{array}{l}\text { Other (skilled care, nursing home, } \\
\text { separate acute care, rehabilitation) }\end{array}$ & $834(9.7)$ & $105(34.2)$ & $321(19.6)$ & $256(10.0)$ & $152(3.7)$ & \\
\hline Readmission w/in 30 days & $364(4.3)$ & $19(6.2)$ & $102(6.3)$ & $116(4.6)$ & $127(3.1)$ & $<0.001$ \\
\hline Not available & 191 & 6 & 34 & 66 & 85 & \\
\hline
\end{tabular}

LOS = length of stay.

Boldface type indicates statistical significance.

Lumbar decompression: cases with primary CPT codes for laminectomy $(63005,63012,63017,63047)$.

* Any minor complication: UTI or superficial SSI.

† Any major complication: cardiac arrest, myocardial infarction, stroke with deficits, wound disruption, deep incisional SSI, organ space SSI, sepsis or septic shock, unplanned intubation, ventilator longer than 48 hours, pneumonia, acute renal failure, progressive renal insufficiency, DVT, pulmonary embolism, or return to operating room.

increased length of stay. In comparison with the younger cohort ( $<65$ years), the other age groups (65-74, 75-84, and $\geq 85$ years) demonstrated incrementally increased likelihoods of nonhome discharge and development of any complication. In comparison with those younger than 65 years, the other 3 age groups had a nearly 3-, 6-, and 14fold, respectively, risk of nonhome discharge. A similar trend was seen with the development of minor complications across the oldest 3 age groups. This is in contrast to 2 prior multicenter studies of lumbar spine surgery that failed to identify a significant difference in complications between the younger than 80-year and the 80-year and older age groups..$^{19,22}$

Our results are consistent with 2 previous studies using national data sets that examined surgical outcomes for older patients with lumbar stenosis, specifically in regard to the outcomes of complications and adverse discharge. A study utilizing the Medicare claims data evaluated a cohort of 32,152 patients with lumbar stenosis, $73 \%$ of whom underwent decompression alone. The fraction of patients discharged to a nursing home and readmitted increased with age: $66-70$ years, $6.6 \%$ discharge to a nursing home and $7.3 \%$ readmitted; $71-74$ years, $9.6 \%$ discharge to a nursing home and $8.1 \%$ readmitted; $75-79$ years, $14.5 \%$ discharge to a nursing home and $9.5 \%$ readmitted; and $\geq$ 80 years, $22.6 \%$ discharge to a nursing home and $11.8 \%$ readmitted. ${ }^{10}$ Another study of the Veterans Affairs National Surgical Quality Improvement Program database examined a cohort of 12,154 lumbar stenosis patients, $85 \%$ of whom underwent lumbar decompression alone, and found age and ASA class to be the best independent predictors, "highest odds," for major medical complications postoperatively. ${ }^{9}$ As in our study, Deyo et al. found that with increasing age an increasing odds of complications occurred. The odds ratios increased incrementally across the 3 comparative age groups: OR 2.31 (60-69 years), OR 3.67 (70-79 years), and OR 4.58 ( $\geq 80$ years). ${ }^{9}$ While both of these studies had a majority of the cohort undergoing lumbar decompression alone, they also included in the analysis patients who underwent simple and complex fusion procedures. Further analysis comparing surgical subgroups in the Medicare claims data revealed 
TABLE 3. Multivariable analysis: outcomes across age groups

\begin{tabular}{ccc}
\hline Outcome \& Age Group & OR $(95 \%$ Cl $)$ & p Value \\
\hline Any minor complication* & & $<0.001$ \\
\hline$\geq 85$ vs $<65$ & $3.47(1.69-7.13)$ & \\
\hline $75-84$ vs $<65$ & $2.34(1.45-3.78)$ & \\
\hline $65-74$ vs $<65$ & $1.44(0.94-2.20)$ & \\
\hline Any major complication* & & 0.291 \\
\hline$\geq 85$ vs $<65$ & $1.31(0.70-2.43)$ & \\
\hline $75-84$ vs $<65$ & $1.33(0.93-1.89)$ & \\
\hline $65-74$ vs $<65$ & $1.31(0.98-1.76)$ & \\
\hline Readmission $\dagger$ & & \\
\hline$\geq 85$ vs $<65$ & $1.35(0.77-2.36)$ & \\
\hline $75-84$ vs $<65$ & $1.52(1.09-2.12)$ & \\
\hline $65-74$ vs $<65$ & $1.24(0.93-1.66)$ & \\
\hline Discharge location (home vs & & \\
nonhome facility) & & \\
\hline$\quad 285$ vs $<65$ & $13.59(9.47-19.49)$ & \\
\hline $75-84$ vs $<65$ & $5.64(4.33-7.34)$ & \\
\hline $65-74$ vs $<65$ & $2.61(2.05-3.12)$ & \\
\hline
\end{tabular}

All models adjusted for race, sex, BMI, hypertension, diabetes, COPD, functional status, smoking status, GFR, hematocrit, platelet count, INR, ASA class, number of levels, and operative time.

Boldface type indicates statistical significance.

${ }^{*} n=8743$. One patient was excluded for missing sex variable.

$\dagger \mathrm{n}=8552$. Several patients were missing readmission information and 1 patient missing sex variable.

$\ddagger n=8626$. Several patients missing discharge information, admitted from a facility, discharged to home facility, died, and 1 missing sex variable.

a significant increased odds of adverse outcomes in those undergoing simple (1- to 2-level) or complex ( $>2$ levels or combined anterior and posterior) fusions. Compared with decompression, patients undergoing simple and complex fusions were nearly 3 times more likely to be discharged to a nursing home. These fusion patients were also nearly 3 times more likely to experience life-threatening complications..$^{10}$ These data argue that the risk of adverse outcomes is correlated with surgical complexity and invasiveness, an argument that has previously been espoused and supported for the elderly population with lumbar stenosis.? Querying the NSQIP Participant Use Data File for specific CPT codes denoting decompressive lumbar procedures allowed us to isolate and refine our cohort. Our analysis does not include fusions and moreover, we controlled for the number of vertebral levels involved in surgery, as a measure of complexity. With our focus on the elderly population and decompression alone, we feel that our data present a unique analysis to add to and enhance the discussion of operative risk and candidacy for elderly patients.

As in other retrospective studies, this cohort analysis carries all the limitations that accompany the retrospective design. While the strengths of NSQIP allow for a highpowered analysis and many advantages over administrative databases, there are limitations. We are limited in defining our cohort solely based on diagnosis and procedural codes. While excellent interrater reliability has been established, error is still possible in this regard. Moreover, although prior operations within 30 days of surgery were excluded, previous lumbar spine surgery preceding that interval was not captured. It is possible that some of our surgeries were revisions; this may act as a confounder. Exclusion of "additional-level" CPTs without the appropriate corresponding initial CPT may have resulted in excluding patients who could have potentially contributed to our analysis; however, this was necessary to control for the extent of surgery though the number of levels. Another limitation of such a multiinstitutional population is the inability to control for hospital variation in procedures and protocols. For example, the ACS Risk Calculator now includes an algorithm for predicting nonhome discharge for certain routine orthopedic procedures; while age is an included factor in the calculation, hospital variation is not. ${ }^{18}$ This appears to be important as shown in a recent analysis that institutions with patients of similar complexity and length of admission had widely varying rates of nonhome discharge..$^{15,28}$ Although we were unable to control for hospital variation, we believe our results remain valid and of clinical importance, supporting and adding to the cumulative literature that the elderly have an increased risk nonhome discharge. Lastly there is an element of selection bias in our cohort as we are evaluating only the elderly patients who are candidates for surgery; these select elderly patients are assessed by spine surgeons as having a favorable risk/benefit ratio for operative intervention. Therefore, the true age-outcomes effect of all patients considered for spine surgery would be suspected to be greater than what we observed, as our study featured only those patients that are deemed to be able to both tolerate and benefit from a procedure.

While previous studies have discussed the correlation of age with adverse outcomes as a reflection of cumulative morbidity that is more likely seen in the elderly population, our analysis controls for several major comorbidities, arguing that age remains an important risk factor for adverse outcomes, even after accounting for major comorbidities. However, surgical candidacy should not be dismissed solely on age. While this study reflects a generalized population, a multitude of factors unique to each patient should be considered during surgical evaluation. In the era of individualized medicine, surgical consultations should consider the overall health and impact of disease to determine whether surgical intervention would be merited and age should definitely play a part in surgical decision making.

\section{Conclusions}

A large, national, multiinstitutional cohort was used to assess short-term outcomes and operative characteristics across elderly patients undergoing lumbar decompression surgery. Increased age was associated with the development of minor complications (UTI and SSI) and discharge to skilled nursing facilities or a rehabilitation unit. While the premorbid status of elderly patients raises concern for potential risk for surgical complications, their health status should not preclude them from a potentially helpful surgical intervention, and each case merits careful indi- 
vidual evaluation in regard to operative candidacy. Discussion of the benefits of pain relief, improved function, and maintaining independence may tip the balance toward favoring operative intervention for both the patient and the surgeon, if both parties agree to incur the potential risks. While age alone should not, a priori, preclude candidacy for surgical intervention, our data provide evidence that, in addition to some comorbidities, age is a predictor for minor complications and nonhome discharge. This study provides discussion points for preoperative counseling important in establishing goals and outlining expectations in regard to lumbar decompression surgery for the elderly population.

\section{Acknowledgments}

This publication was made possible by funding from the Mayo Clinic Robert D. and Patricia E. Kern Center for the Science of Health Care Delivery.

\section{References}

1. Aalto TJ, Malmivaara A, Kovacs F, Herno A, Alen M, Salmi $\mathrm{L}$, et al: Preoperative predictors for postoperative clinical outcome in lumbar spinal stenosis: systematic review. Spine (Phila Pa 1976) 31:E648-E663, 2006

2. Amundsen T, Weber H, Nordal HJ, Magnaes B, Abdelnoor M, Lilleâs F: Lumbar spinal stenosis: conservative or surgical management?: A prospective 10-year study. Spine (Phila Pa 1976) 25:1424-1436, 2000

3. Atlas SJ, Keller RB, Wu YA, Deyo RA, Singer DE: Longterm outcomes of surgical and nonsurgical management of lumbar spinal stenosis: 8 to 10 year results from the Maine Lumbar Spine Study. Spine (Phila Pa 1976) 30:936-943, 2005

4. Balabaud L, Pitel S, Caux I, Dova C, Richard B, Antonietti $\mathrm{P}$, et al: Lumbar spine surgery in patients 80 years of age or older: morbidity and mortality. Eur J Orthop Surg Traumatol 25 (Suppl 1):S205-S212, 2015

5. Best NM, Sasso RC: Outpatient lumbar spine decompression in 233 patients 65 years of age or older. Spine (Phila Pa 1976) 32:1135-1140, 2007

6. Carreon LY, Puno RM, Dimar JR II, Glassman SD, Johnson JR: Perioperative complications of posterior lumbar decompression and arthrodesis in older adults. J Bone Joint Surg Am 85-A:2089-2092, 2003

7. Cassinelli EH, Eubanks J, Vogt M, Furey C, Yoo J, Bohlman $\mathrm{HH}$ : Risk factors for the development of perioperative complications in elderly patients undergoing lumbar decompression and arthrodesis for spinal stenosis: an analysis of 166 patients. Spine (Phila Pa 1976) 32:230-235, 2007

8. Ciol MA, Deyo RA, Howell E, Kreif S: An assessment of surgery for spinal stenosis: time trends, geographic variations, complications, and reoperations. J Am Geriatr Soc 44:285-290, 1996

9. Deyo RA, Hickam D, Duckart JP, Piedra M: Complications after surgery for lumbar stenosis in a veteran population. Spine (Phila Pa 1976) 38:1695-1702, 2013

10. Deyo RA, Mirza SK, Martin BI, Kreuter W, Goodman DC, Jarvik JG: Trends, major medical complications, and charges associated with surgery for lumbar spinal stenosis in older adults. JAMA 303:1259-1265, 2010

11. Fehlings MG, Tetreault L, Nater A, Choma T, Harrop J, Mroz $\mathrm{T}$, et al: The aging of the global population: the changing epidemiology of disease and spinal disorders. Neurosurgery 77 (Suppl 4):S1-S5, 2015

12. Galiano K, Obwegeser AA, Gabl MV, Bauer R, Twerdy K:
Long-term outcome of laminectomy for spinal stenosis in octogenarians. Spine (Phila Pa 1976) 30:332-335, 2005

13. Gruskay JA, Fu M, Bohl DD, Webb ML, Grauer JN: Factors affecting length of stay after elective posterior lumbar spine surgery: a multivariate analysis. Spine J 15:1188-1195, 2015

14. Hu SS, Holly EA, Lele C, Averbach S, Kristiansen J, Schiff $\mathrm{M}$, et al: Patient outcomes after spinal reconstructive surgery in patients $>$ or $=40$ years of age. J Spinal Disord 9:460469, 1996

15. Hyder JA: Genuflecting to hospital variation in home discharge. JAMA Surg 150:1203, 2015 (Letter)

16. Kalbarczyk A, Lukes A, Seiler RW: Surgical treatment of lumbar spinal stenosis in the elderly. Acta Neurochir (Wien) 140:637-641, 1998

17. McCutcheon BA, Ubl DS, Babu M, Maloney P, Murphy M, Kerezoudis P, et al: Predictors of surgical site infection following craniotomy for intracranial neoplasms: an analysis of prospectively collected data in the ACS-NSQIP database. World Neurosurg 88:350-358, 2016

18. Mohanty S, Liu Y, Paruch JL, Kmiecik TE, Cohen ME, Ko CY, et al: Risk of discharge to postacute care: a patientcentered outcome for the American College Of Surgeons National Surgical Quality Improvement Program surgical risk calculator. JAMA Surg 150:480-484, 2015

19. Nanjo Y, Nagashima H, Dokai T, Hamamoto Y, Hashiguchi $\mathrm{H}$, Ishii $\mathrm{H}$, et al: Clinical features and surgical outcomes of lumbar spinal stenosis in patients aged 80 years or older: a multi-center retrospective study. Arch Orthop Trauma Surg 133:1243-1248, 2013

20. Raffo CS, Lauerman WC: Predicting morbidity and mortality of lumbar spine arthrodesis in patients in their ninth decade. Spine (Phila Pa 1976) 31:99-103, 2006

21. Ragab AA, Fye MA, Bohlman HH: Surgery of the lumbar spine for spinal stenosis in 118 patients 70 years of age or older. Spine (Phila Pa 1976) 28:348-353, 2003

22. Rihn JA, Hilibrand AS, Zhao W, Lurie JD, Vaccaro AR, Albert TJ, et al: Effectiveness of surgery for lumbar stenosis and degenerative spondylolisthesis in the octogenarian population: analysis of the Spine Patient Outcomes Research Trial (SPORT) data. J Bone Joint Surg Am 97:177-185, 2015

23. Rosen DS, O'Toole JE, Eichholz KM, Hrubes M, Huo D, Sandhu FA, et al: Minimally invasive lumbar spinal decompression in the elderly: outcomes of 50 patients aged 75 years and older. Neurosurgery 60:503-510, 2007

24. Shamji MF, Mroz T, Hsu W, Chutkan N: Management of degenerative lumbar spinal stenosis in the elderly. Neurosurgery 77 (Suppl 4):S68-S74, 2015

25. Shiloach M, Frencher SK Jr, Steeger JE, Rowell KS, Bartzokis K, Tomeh MG, et al: Toward robust information: data quality and inter-rater reliability in the American College of Surgeons National Surgical Quality Improvement Program. J Am Coll Surg 210:6-16, 2010

26. Silverstein MP, Miller JA, Xiao R, Lubelski D, Benzel EC, Mroz TE: The impact of diabetes upon quality of life outcomes after lumbar decompression. Spine J 16:714-721, 2016

27. Tadokoro K, Miyamoto H, Sumi M, Shimomura T: The prognosis of conservative treatments for lumbar spinal stenosis: analysis of patients over 70 years of age. Spine (Phila Pa 1976) 30:2458-2463, 2005

28. Tomek IM, Sabel AL, Froimson MI, Muschler G, Jevsevar DS, Koenig KM, et al: A collaborative of leading health systems finds wide variations in total knee replacement delivery and takes steps to improve value. Health Aff (Millwood) 31:1329-1338, 2012

29. United Nations: World Population Ageing 1950-2050. New York: United Nations, 2001 (http://www.un.org/esa/ population/publications/worldageing19502050/) [Accessed September 16, 2016] 


\section{Disclosures}

The authors report no conflict of interest concerning the materials or methods used in this study or the findings specified in this paper.

\section{Author Contributions}

Conception and design: Bydon, Murphy, Maloney, McCutcheon, Rinaldo, Kerezoudis. Acquisition of data: McCutcheon, Ubl. Analysis and interpretation of data: Bydon, Murphy, Maloney, Ubl, Habermann. Drafting the article: Murphy, Gilder, Shepherd, Kerezoudis. Critically revising the article: all authors. Reviewed submitted version of manuscript: all authors. Approved the final version of the manuscript on behalf of all authors: Bydon. Statistical analysis: Ubl, Crowson, Habermann. Administrative/techni- cal/material support: Bydon. Study supervision: Bydon, Krauss, Habermann.

\section{Supplemental Information}

Previous Presentations

Portions of this work were presented in poster presentation formats at the 2016 AANS Annual Scientific Meeting, April 30 to May 4, 2016, in Chicago, Illinois, as well as at the Mayo Clinic Young Investigator Research Symposium, March 19, 2016, in Rochester, Minnesota.

\section{Correspondence}

Mohamad Bydon, Department of Neurologic Surgery, Mayo Clinic, 200 First St. SW, Rochester, MN 55905. email: bydon. mohamad@mayo.edu. 